\title{
New evaluation of general purpose neutron data for stable W-isotopes up to $200 \mathrm{MeV}$
}

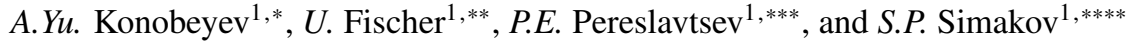 \\ ${ }^{1}$ Karlsruhe Institute of Technology (KIT) Institute for Neutron Physics and Reactor Technology Hermann-von-Helmholtz-Platz 176344 \\ Eggenstein-Leopoldshafen Germany
}

\begin{abstract}
In the frame of the Power Plant Physics and Technology of EUROfusion, new evaluations of general purpose neutron cross-section data were performed for the ${ }^{180,182,183,184,186} \mathrm{~W}$ isotopes covering the neutron energy up to $200 \mathrm{MeV}$. A special version of the TALYS nuclear model code implementing the geometry dependent hybrid model supplied with models for the non-equilibrium cluster emission was applied for calculations of the nuclide production and the energy distribution of the emitted particles. The parameters of the GDH model were properly estimated using measured data for individual tungsten isotopes. The neutron cross-sections were evaluated making use of available experimental data, systematics including estimated A-dependence of components of gas production cross-sections, and covariance information produced as part of the evaluation process. The BEKED code package, developed at KIT, was applied for calculations of co-variances using a dedicated Monte Carlo method. The evaluated data were processed into standard ENDF data format using the TEFAL code and the FOX module of the BEKED system. The evaluated data files were checked for errors and inconsistencies, processed with the NJOY code into ACE data format, and benchmarked against available integral experiments with MCNP neutron transport calculations.
\end{abstract}

\section{Introduction}

Due to its unique properties, tungsten is a prime candidate for the first wall in fusion devices [1]. Reliable, highquality evaluated data for tungsten are of particular importance for modelling neutron irradiation.

It has been more than ten years since evaluating data for tungsten isotopes in KIT [2]. The data are included in the JEFF-3.3 [3] and were successfully used for neutron transport calculations for fusion and fission reactors. Since then, calculation and evaluation methods have been further developed, and new experimental data have appeared.

The aim of this work was to use new information and advanced methods to obtain evaluated data for stable tungsten isotopes with atomic mass number 180, 182, 183, 184 , and 186 irradiated with neutrons. The new data evaluation takes into account the experience of using previous data and provides both further improvement of the data quality concerning reliability and completeness. The calculation of nuclide production cross-sections and particle energy distributions was performed using a special version of the TALYS code [4], [5], [6], [7] providing calculations with geometry dependent hybrid model (GDH) [8], [9],[10], [11].

Section 2 describes the evaluation procedure and Section 3 presents several examples of obtained data for tungsten isotopes.

\footnotetext{
*e-mail: alexander.konobeev@kit.edu

**e-mail: ulrich.fischer@kit.edu

***e-mail: pavel.pereslavtsev@kit.edu

****e-mail: stanislav.simakov@ partner.kit.edu
}

\section{Evaluation procedure}

The evaluation consists of following steps: i) the calculation of cross-sections, angular distributions for elastic scattering, energy distributions of emitted particles, and the calculation of covariance matrices for cross-sections, ii) the processing obtained data in a file in the ENDF-6 format [12], iii) the selection and analysis of experimental data for subsequent combination with results of calculations, iv) the evaluation using experimental data, results of model calculations, and covariance information, v) the recording final data in the ENDF-6 format, and vi) the general check of evaluated data file. The steps are briefly discussed below.

\subsection{Calculations using nuclear models}

The special version of the TALYS code [5], [6] implementing GDH model [9] and supplied with models for the non-equilibrium cluster emission [11], [6] was applied for calculations of nuclear reaction cross-sections and particle energy distributions. The parameters of the GDH model were properly estimated using available measured data [7].

The energy distributions of pre-equilibrium deuterons, tritons, ${ }^{3} \mathrm{He}$-nuclei, and $\alpha$-particles are calculated as a sum of components concerning pick-up, knock-out, and direct processes. Details are discussed in Refs. [11], [6], [7].

An extensive comparison of model predictions with experimental data is can be found in Ref.[7].

The total cross-section, elastic cross-section, elastic angular distribution, and contribution of direct processes 
in inelastic scattering were calculated using the ECIS-06 code [13] integrated in the TALYS code [5].

The equilibrium particle emission was simulated using the Hauser-Feshbach model [4], [14]. The Fermi gas model with the energy dependent level density parameter [15] combined with the "constant temperature" model [4], [16] was applied for nuclear level density calculations.

The covariance matrices for cross-sections were calculated using the Monte Carlo method proposed in Ref.[17]. The calculation contains the following steps: the choice of the best set of parameters for selected nuclear models, the assessment of uncertainties of model parameters, the Monte Carlo sampling of input data sets, the execution of calculations for obtained input data files, and the calculation of covariance matrices for particular reactions.

\subsection{Processing of output data}

Processing of TALYS output information and recording of preliminary data file in the ENDF-6 format was performed using the TEFAL-1.9 code [18],[19]. The code collects TALYS output data files prepared with the input option "endf y".

At this stage, the resonance parameters from JEFF-3.3 were included in files for ${ }^{182} \mathrm{~W},{ }^{183} \mathrm{~W},{ }^{184} \mathrm{~W}$, and ${ }^{186} \mathrm{~W}$,

\subsection{Use of experimental data}

Experimental data were taken from a number of works compiled in EXFOR [20] and cited in Ref.[21] and [22]. Data were converted to $\mathrm{C} 4$ format [23] for further use. In some cases, errors of cross-sections, automatically selected by processing, were specified using available information.

The ENSDF data [24] were used for the analysis of measurements of $\left(\mathrm{n}, \mathrm{n}^{\prime}\right)$ reaction cross-sections.

\subsection{Use of systematics}

Cross-sections measured in [25] for ${ }^{182} \mathrm{~W},{ }^{184} \mathrm{~W}$, and ${ }^{186} \mathrm{~W}$ were used to estimate the total reaction cross-section for isotopes ${ }^{180} \mathrm{~W}$ and ${ }^{183} \mathrm{~W}$ [22]. The estimation was made applying the method of Ref.[26] basing on the parameterization of a large number of experimental data.

The data from Ref.[27] were applied for the correction of calculated total light charge particle production crosssections. Data [27] are the result of the evaluation of the atomic mass number dependency of corresponding cross sections at fixed neutron incident energy. See details in Refs.[27],[28].

\subsection{Cross-section evaluation}

The evaluation of cross-sections using experimental data, results of model calculations, and covariance information was performed using the generalized least-squares method [29]. The BEKED package [30] was applied for numerical computations.

\subsection{Recording data in ENDF-6 format}

Evaluated data were consistently integrated in the final data file.

Even a relative small change in the cross-section for a specific reaction after the evaluation comparing to calculated value results to specific changes in the final data file: change of the contribution of isomers $(\mathrm{MF}=9,10)$, absorption cross section $(\mathrm{MF} / \mathrm{MT}=3 / 3)$, total cross section, sum of cross sections recorded in $\mathrm{MF} / \mathrm{MT}=3 / 5$, the production cross-section for corresponding residual nucleus in $\mathrm{MF} / \mathrm{MT}=6 / 5$, the yields for all residuals recorded in the $\mathrm{MF} / \mathrm{MT}=6 / 5$, including neutron production, gas-production components, and $\gamma$-production due to the change in $\mathrm{MF} / \mathrm{MT}=3 / 5$. Such re-calculations and corrections of data were performed using the FOX code from BEKED. Special attention was paid to avoiding jumps in cross sections values at the transition from $\mathrm{MF}=3$ to $\mathrm{MF} / \mathrm{MT}=6 / 5$ data representation.

Checking the obtained file before processing with NJOY [31] is carried out using checking codes [32] and the COVEIG code [33].

\section{Evaluated data}

The section presents several illustrations of the data obtained, showing typical agreement with experimental data and differences with other evaluations.

A comprehensive comparison of evaluated data and the data of other authors can be found in Ref.[21] and [22].

Figures 1 and 2 show the evaluated total cross-section for ${ }^{183} \mathrm{~W}$ and ${ }^{186} \mathrm{~W}$, data from different libraries, experimental data, and cross-sections derived using measurements [25] for other tungsten isotopes. Obtained data are in good agreement with available experimental information.

Examples of evaluated $(\mathrm{n}, 2 \mathrm{n})$ reaction cross-sections are shown in Figs.3,4. Figures 5,6 illustrates $(n, p)$ reaction cross-sections.

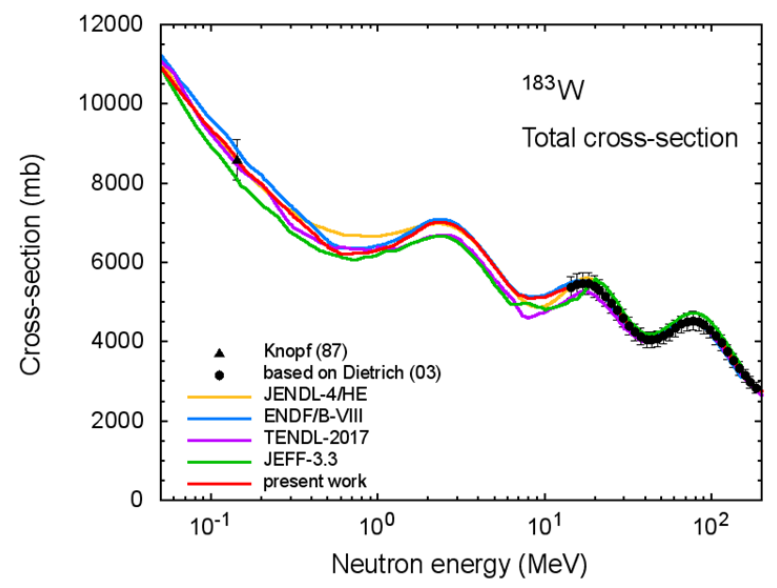

Figure 1. The total reaction cross-section for neutron irradiation of ${ }^{183} \mathrm{~W}$ evaluated in the present work, measured data, and data taken from different libraries. Experimental data on this and all other figures are cited on Refs.[21],[22]. 


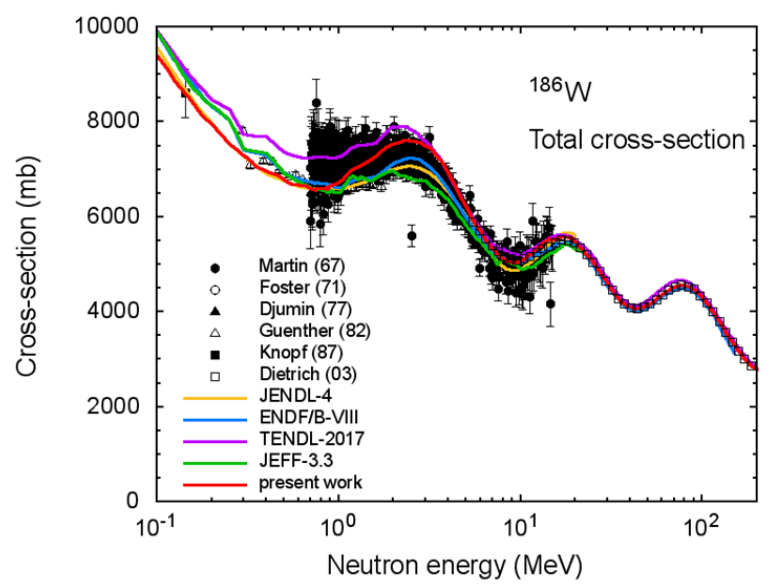

Figure 2. The same as in Fig.1 but for ${ }^{186} \mathrm{~W}$.

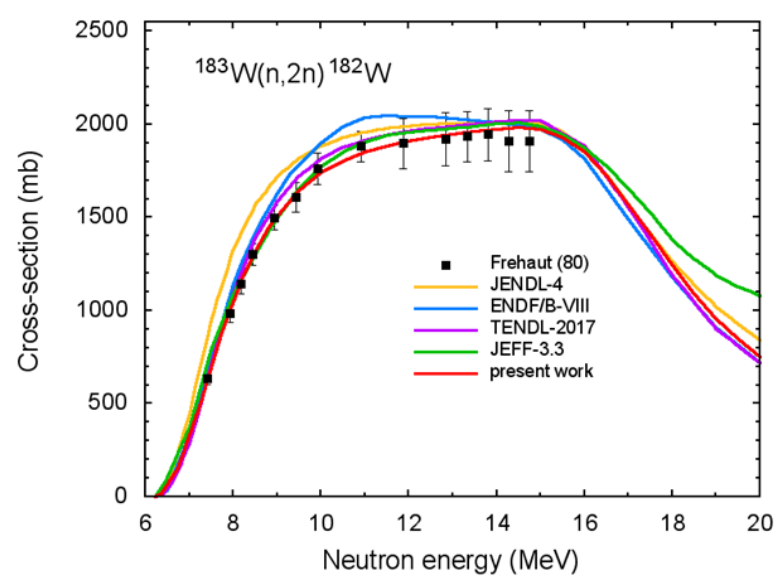

Figure 3. The ${ }^{183} \mathrm{~W}(\mathrm{n}, 2 \mathrm{n}){ }^{182} \mathrm{~W}$ reaction cross-section.

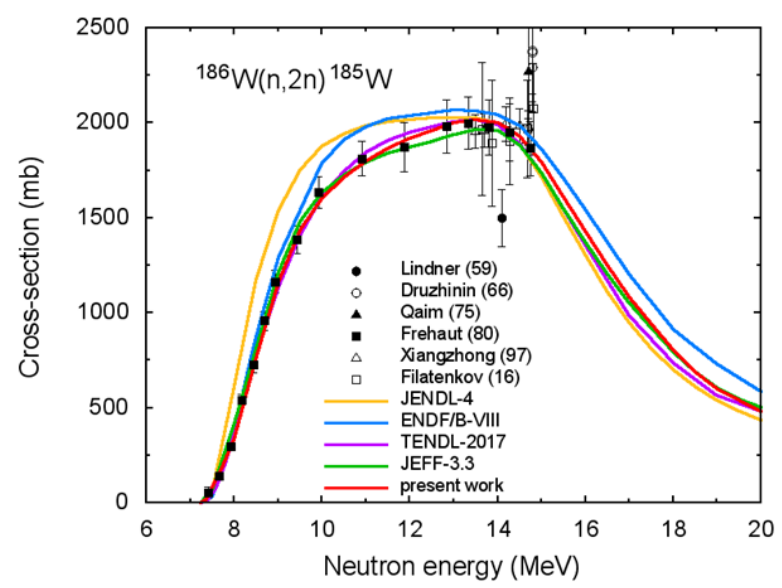

Figure 4. The ${ }^{186} \mathrm{~W}(\mathrm{n}, 2 \mathrm{n}){ }^{185} \mathrm{~W}$ reaction cross-section.

\section{Conclusion}

New general purpose data files were prepared for stable tungsten isotopes $180,182,183,184,186 \mathrm{~W}$ isotopes at primary neutron energy up to $200 \mathrm{MeV}$. A special version of the TALYS code implementing the geometry dependent hy-

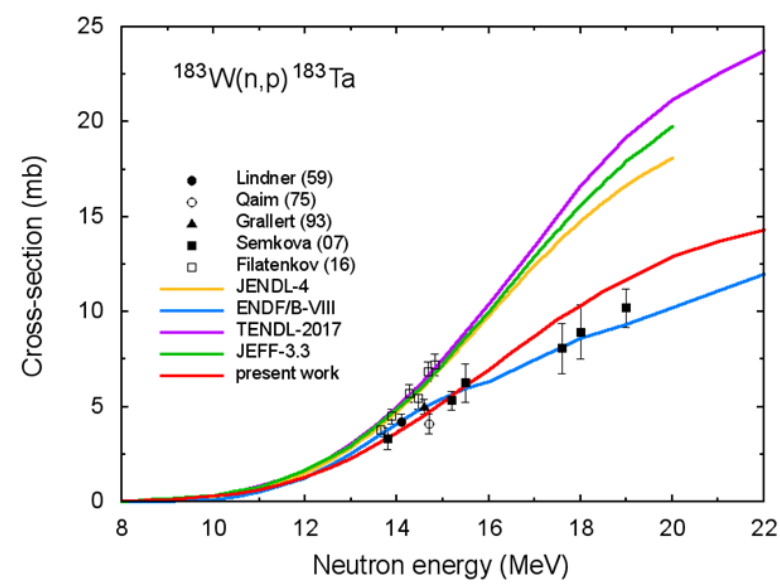

Figure 5. The ${ }^{183} \mathrm{~W}(\mathrm{n}, \mathrm{p})^{183}$ Ta reaction cross-section.

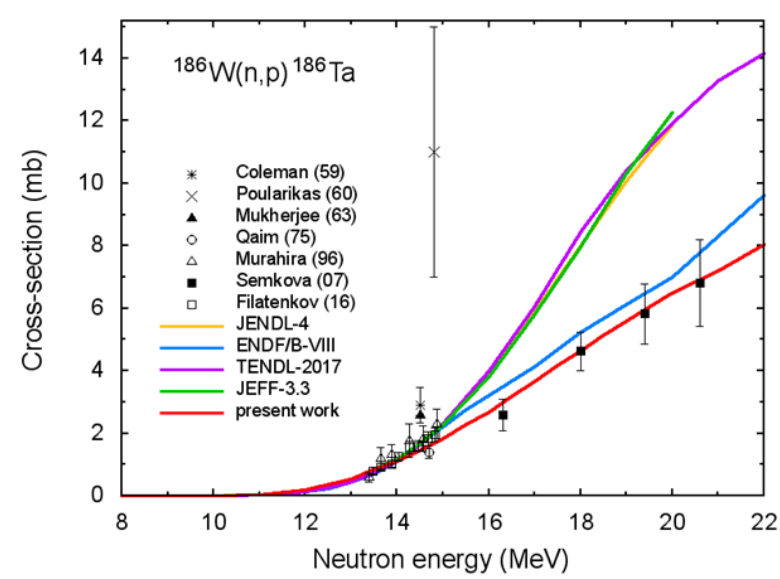

Figure 6. The ${ }^{186} \mathrm{~W}(\mathrm{n}, \mathrm{p})^{186} \mathrm{Ta}$ reaction cross-section.

brid model was applied for calculations of nuclide production and particle energy distributions. The evaluation of cross-sections was performed using available experimental data, systematics including estimated A-dependence of components of gas production cross-sections, and obtained covariance information.

Detail comparison of obtained data with measurements and data from evaluated data libraries is presented in Refs.[21],[22].

\section{Acknowledgment}

This work has been carried out within the framework of the EUROfusion Consortium and has received funding from the Euratom research and training programme 2014-2018 and 2019-2020 under grant agreement No 633053. The views and opinions expressed herein do not necessarily reflect those of the European Commission.

\section{References}

[1] E. Bernard, R. Sakamoto, A. Kreter, M.F. Barthe, E.Autissier, P. Desgardin, H. Yamada, S. GarciaArgote, G. Pieters, J. Chene, B. Rousseau, C. Grisolia, 
Tungsten as a plasma-facing material in fusion devices: impact of helium high-temperature irradiation on hydrogen retention and damages in the material, Physica Scripta T170, 014023 (2017)

[2] P. Pereslavtsev, U. Fischer, Evaluation of $n+W$ cross section data up to $150 \mathrm{MeV}$ neutron energy, AIP Conference Proceedings 769, 215 (2005); https://doi.org/10.1063/1.1944993

[3] The Joint Evaluated Fission and $\mathrm{Fu}-$ sion File. JEFF-3.3, http://www.oecdnea.org/dbdata/jeff/jeff33/index.html

[4] A.J. Koning, S. Hilaire and M.C. Duijvestijn, TALYS1.0, Proc. International Conference on Nuclear Data for Science and Technology, April 22-27, 2007, Nice, France, editors O. Bersillon, F. Gunsing, E. Bauge, R. Jacqmin, and S. Leray, EDP Sciences, 2008, p. 211

[5] A.J. Koning, S. Hilaire, M. Duijvestijn, TALYS-1.7, A nuclear reaction program, Nuclear Research and Consultancy Group (NRG), May 7, 2015

[6] A.Yu. Konobeyev, U. Fischer, A.J. Koning, P.E. Pereslavtsev, M. Blann, Implementation of the geometry dependent hybrid model in TALYS, J. Korean Physical Society 59, 935 (2011)

[7] A.Yu. Konobeyev, U. Fischer, P.E. Pereslavtsev, A. Koning, M. Blann, Implementation of GDH model in TALYS-1.7 code, KIT Scientific Working Papers 45, 2016; https://publikationen.bibliothek.kit.edu/1000052543

[8] M. Blann, Importance of the nuclear density distribution on pre-equilibrium decay, Phys. Rev. Lett. 28, 757 (1972)

[9] M. Blann, H.K. Vonach, Global test of modified precompound decay models, Phys. Rev. C 28, 1475 (1983)

[10] M. Blann, ALICE-91: Statistical model code system with fission competition, RSIC Code Package PSR146,1991

[11] C.H.M. Broeders, A.Yu. Konobeyev, A.Yu. Korovin, V.P. Lunev, M. Blann, ALICE/ASH - Pre-compound and evaporation model code system for calculation of excitation functions, energy and angular distributions of emitted particles in nuclear reactions at intermediate energies, Report FZKA 7183, May, 2006, http://bibliothek.fzk.de/zb/berichte/FZKA7183.pdf

[12] ENDF-6 formats manual, Report BNL-90365-2009 Rev.2, Ed. A. Trkov, M. Herman and D. A. Brown, October 24, 2012

[13] J. Raynal, ECIS-06: Code system to solve coupled differential equations arising in nuclear model calculations, RSICC PSR-227, 2007

[14] W. Hauser, H. Feshbach, The inelastic scattering of neutrons, Phys. Rev. 87, 366 (1952)

[15] A.V. Ignatyuk, G.N. Smirenkin, A.S. Tishin, Phenomenological description of the energy dependence of the level density parameter, Sov. J. Nucl. Phys. 21, 255 (1975)

[16] A. Gilbert, A.G.W. Cameron, A composite nuclearlevel density formula with shell corrections, Can. J. Phys. 43, 1446 (1965)
[17] D.L. Smith, Covariance matrices for nuclear cross sections derived from nuclear model calculations, ANL/NDM-159, Argonne National Laboratory, 2004

[18] A.J. Koning, D. Rochman, Modern nuclear data evaluation with the TALYS code system, Nucl. Data Sheets 113, 2841 (2012)

[19] A.J. Koning, TEFAL-1.9: Making nuclear data libraries using TALYS, Nuclear Research and Consultancy Group (NRG), November 2017

[20] Experimental Nuclear Reaction Data (EXFOR), https://www-nds.iaea.org/exfor/exfor.htm

[21] A.Yu. Konobeyev, U. Fischer, P.E. Pereslavtsev, S.P. Simakov, Evaluated data files for neutron irradiation of ${ }^{182} \mathrm{~W}$ and ${ }^{186} \mathrm{~W}$ at energies up to 200 MeV, KIT Scientific Working Papers 108, 2019; https://publikationen.bibliothek.kit.edu/1000090132

[22] A.Yu. Konobeyev, U. Fischer, P.E. Pereslavtsev, S.P. Simakov, Evaluated data files for $n+{ }^{180} \mathrm{~W}$ and ${ }^{183} \mathrm{~W}$ irradiation at incident neutron energies up to $200 \mathrm{MeV}$, KIT Scientific Working Papers 123, 2019; https://publikationen.bibliothek.kit.edu/1000096730

[23] D.E. Cullen, A. Trkov, Computational format C4, https://www-nds.iaea.org/nrdc/basics/exfor-basics3.html

[24] ENSDF: Evaluated Nuclear Structure Data File Search and Retrieval, https://www. nndc.bnl.gov/ensdf/EnsdfDispatcherServlet

[25] F.S. Dietrich, J.D. Anderson, R.W. Bauer, S.M. Grimes, R.W. Finlay, W.P. Abfalterer, F.B. Bateman, R.C. Haight, G.L. Morgan, E. Bauge, J.P. Delaroche, P. Romain, Importance of isovector effects in reproducing neutron total cross section differences in the $\mathrm{W}$ isotopes, Phys. Rev. C 67, 044606 (2003)

[26] V.S. Barashenkov, A. Polanski, Electronic Guide for Nuclear Cross-Sections, Joint Institute for Nuclear Research Communication E2-94-417, JINR, Dubna, Russia, 1994.

[27] A.Yu. Konobeyev, U. Fischer, Complete gas production data library for nuclides from $\mathrm{Mg}$ to $\mathrm{Bi}$ at neutron incident energies up to $200 \mathrm{MeV}$, Report KIT SWP 36 (2015), http://digbib.ubka.unikarlsruhe.de/volltexte/1000049466

[28] A.Yu. Konobeyev, U. Fischer, Reference data for evaluation of gas production cross-sections in proton induced reactions at intermediate energies, KIT SR 7660 (2014), www.ksp.kit.edu/download/1000038463

[29] D.L. Smith, A Least-squares computational tool kit, ANL/NDM-128, Argonne National Laboratory, 1993

[30] A.Yu. Konobeyev, U. Fischer, P.E. Pereslavtsev, Computational approach for evaluation of nuclear data including covariance information, J. Kor. Phys. Soc. 59, 923 (2011)

[31] The NJOY Nuclear data processing system, version 2016, https://www. njoy21.io/NJOY2016/

[32] ENDF-6 Checking and Utility Codes, https://wwwnds.iaea.org/public/endf/utility/

[33] A. Trkov, Program COVEIG, https://wwwnds.iaea.org/IRDFF/coveig.for 